\title{
Space Junk Cleaning Strategy and Portfolio Optimization Modeling
}

\author{
Yanlin Ma and Wuyue Yang* \\ No.35 Qinghuadong Road, Haidian Dist, Beijing Forestry University, Beijing, China \\ ${ }^{*}$ Corresponding author
}

\begin{abstract}
This essay firstly analyzed the development trend of space debris, and the establishment of a development model of low-orbit space debris size. On this basis, this essay in-depth analysis of the efficiency of different methods to eliminate debris. The relational model is built for the elimination of space debris each way and cost efficiency, compare and contrast the applicability of the different methods. Finally, we analyzed separately from a risk, cost, and risk appetite three angles. We are considered different situations to build combinatorial optimization model made a different way to eliminate space debris, which could optimize the choice under different restrictions.
\end{abstract}

Keywords- component; space debris; portfolio optimization

\section{INTRODUCTION}

As the world aerospace level of progress, every year a large number of flight equipment into space. Inevitably stranded a lot of space debris. Space debris on satellites and other equipment caused great damage. How to eliminate the interference of space equipment space debris has become an issue that must be considered in the field of aerospace. Therefore, it is necessary to eliminate space debris research ways. This essay deeply explores the present situation and development of space debris. The mathematical model is built in aspect of cost, benefits, and risk in comparison of different methods about cleaning up space debris applicability and determining solution or method of combination and whether there have commercial opportunity.

\section{The Number of Space Debris Growth Model}

\section{A. The definition of Space Debris}

Space debris refers to any elder than one year old objects which can enter the outside space. The debris run at the area named LEO which is off the ground between 3500 1800 km road area, That is centered geocentric, $6728 \mathrm{~km}$ radius area and $8178 \mathrm{~km}$ between the two layers of spherical shells. For space particle, we know the following pieces of information.

\section{B. Characteristics of Space Debris}

Space debris qualified "box particle", an increase of space debris and reduce the coefficient of a differential equation can be expressed by physical parameters.

Moving space debris disobedience movement law of ideal gas molecules.

Space debris within the region of movement in any direction is free, random, until the debris collide with another stop.

\section{Source of Space Debris}

Objects launched into space;The explore of space objects;Collision of debris

\section{Decrease of Space Debris}

Fell by atmospheric drag;Fell by recycle;Fell by specific machine

\section{E. Changes of Space Debris}

For the change of space debris, we can be quantified as $N$ (annual rate of change) $N=\frac{d N}{d t}$ where $N$ is the total number of space debris, $t$ within the track area at any time (in years).

Factors causing changes in the number of space debris, mainly in the following:

Contribution launch into orbit of space debris objects onyear rate of change $N_{1}$;Orbit space objects explode, fragmentation contribution to the annual rate of change debris $N_{2}$; Debris Collision contribution to the rate of change in the debris $N_{3}$; Contribution of atmospheric drag on the rate of change in the debris $N_{4}$; Regularly collect objects on the contribution of space debris-year rate of change $N_{5}$; Private collector collects contributions to the rate of change in the debris.

Since the number of times per year recovered spacecraft from orbit very little in the near future we can not have a dedicated collector of debris, so we can make: $N_{5}=N_{6}=0$.

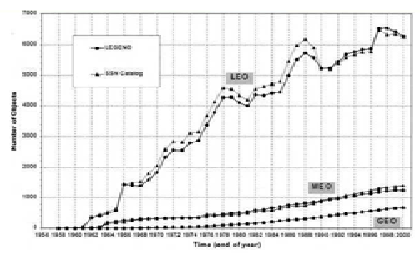

FIGURE I. FIGURE QUOTE FROM J.-C. LIOU[7]

As the data shown in the picture, we figured out 10 dots for our analysis formula and the result is below:

According to our fitting model, coefficient can be decided in the debris development model as: $-14 N^{2}+665.1 N-1714.8$

The result discussion:

As the data we got ,two kinds of situation and their tableau can be described below: 
1) Small fragment: According to the data, we can suppose the development of debris obey the rule of memoryless property, so the fitting curve is:

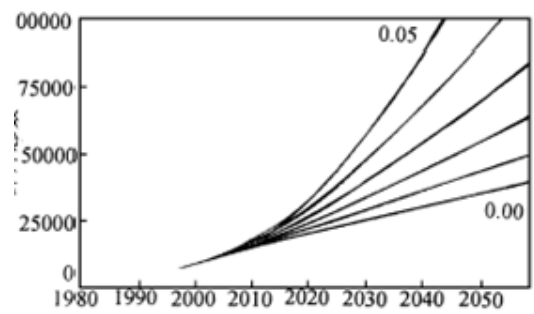

FIGURE II. FIGURE QUOTE FROM ZHU XIAOYI[5].

2) Large fragment

$$
y=-78.1614+1.0448 \mathrm{e}^{0.0439 t}
$$

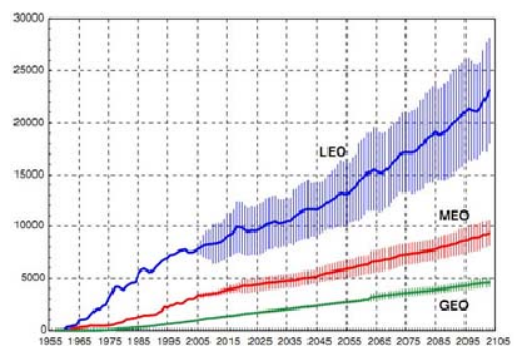

FIGURE III. FIGURE QUOTE FROM J.-C. LIOU[6]

$$
y=-13.6513+1.0013 \mathrm{e}^{0.0113 \mathrm{t}}
$$

When the initial debris fewer and fewer collisions between cosmic. At this small base pieces, the growth is very slow, after slowly increase as the debris, increasing the number of collisions. When the number of fragments exponential upward trend, this is the fragmentation of natural growth over time. Compared to the small pieces of debris large increase more slowly, because more difficult to generate a large fragment. Large debris generation process also will produce small pieces.

By studying the size of the debris growth, follow-up will further explore ways to eliminate debris, Better debris elimination optimization process.

\section{Method of Remove Space Debris And Model ANALYSIS}

\section{A. Earth-Based Repetitively Pulsed Laser}

1) Overview: Existing laser space junk clean-up system uses a ground-based laser system LISP and active beam phase correction beam director and computer-intensive, highresolution optical detection system combine to within a range of 500 kilometers in diameter small to $1 \mathrm{~cm}$ of object orientation. Positioning the object is repeated pulsating laser irradiation, so that the object is positioned ablation derailed. $20 \mathrm{KW}$ average laser and the most advanced monitoring technologies to clear a height $1000 \mathrm{~km}$ of more than $1 \mathrm{~cm}$ in diameter near-Earth orbit space debris, less than $100 \mathrm{~kg}$ can run a large number of fragments of four years, the diameter of $1 \mathrm{~cm} \sim 20 \mathrm{~cm}$ of space debris would be continuous run for two years. The method with reasonable laser and detection system to eliminate 300,000 space debris in four years.

The entire system consists of a moderate average power consumption of repetitive pulse laser, astronomical observatories style guide and the capture and tracking systems.

2) Laser: We use C.R. Phipps 'Laser Impulse Space Propulsion' system as the research object[1]. laser wavelength is selected to be $530 \mathrm{~nm}$, since the strength of such a pulse can ensure the reasonableness of atmospheric transport and ensure a low cost.

At the same time we need to laser spot in the maximum range of $1400 \mathrm{~km}$ domestic spot with a diameter of $40 \mathrm{~cm}$. Laser pulse of energy optimization $\mathrm{W}$ in consideration the cost of capital and defragmentation speed selection was $\mathrm{W}=20 \mathrm{~kJ}$. Also select the laser average power $\mathrm{P}=20 \mathrm{KW}$.

For laser pulses for each target, we need to consider different situations. For debris altitude $\mathrm{h}<1000 \mathrm{~km}$ of track, $\Delta \mathrm{v} \mid \leqslant 235 \mathrm{~m} / \mathrm{s}$ is sufficient when the security situation in the target object reentry ablation; while the track $1000 \mathrm{~km} *$ $500 \mathrm{~km}$ case, $|\Delta \mathrm{v}|=113 \mathrm{~m} / \mathrm{s}$ is sufficient. The objective was to take into account the near-Earth orbit Chen This is $\$ 20 \mathrm{k} /$ $\mathrm{kg}$, ground-based lasers are most suitable.

Since in vacuum, the opaque material is "light absorbing surface material", for the presence of this material one of the most suitable pulse intensity $I_{o p t}$, selection threshold vicinity thereof, so that the maximum $\mathrm{Cm}$, reason for this choice is that in $I_{o p t}$ or less, more and more of the laser energy is invested in heating and melting rather than in ablation, while above $I_{o p t}$ the behavior $C_{m} \propto \frac{1}{v} \propto \frac{1}{(I \lambda \sqrt{\tau})^{\frac{1}{4}}}$ dominates. Space debris $\Delta \mathrm{v}$ maximum possible total energy $\mathrm{W}$ $=9.4 \mathrm{~kJ} / \mathrm{g}$ of the laser pulse is, in this case, only a small portion of the object will be ablated, single beam of laser energy required relationship can They are listed as follows: $W=\frac{u M_{0}}{n \eta C_{m}}|\Delta v|$

$$
\text { Where } u=\left(\frac{d_{s}}{d}\right)^{2} d_{s}>d=1 d_{s} \leq d
$$

where by we obtain $\Delta \mathrm{v}$ laser pulses generated total demand of expression: $n=5.0 \times 10^{-5} \frac{u M_{0}|\Delta v|}{C_{m} A_{5} \sqrt{\tau}}$

3) Detection and tracking devices: Detection and tracking device consists of an optical detection system through efficiency and baseline detection system components, the entire detection system includes a telescope, brackets, electronic detectors and digital acquisition system, the entire device unit price is $\$ 200,000$, can be a lot of copy, these detectors can select install several sites along the equator, but there are some flaws, effective use of time these detectors is 3 to 4 hours per day primarily to detect space debris altitude of 
$500 \mathrm{~km} \sim 1000 \mathrm{~km}$, the trace output mode can be divided into coarse $(1.06 \mu \mathrm{m}, 500 \mathrm{~J})$ and fine $(530 \mathrm{~nm}, 400 \mathrm{~J})$. If we have to remove pieces of debris $300 \mathrm{k}$ in four years time, we will have enough time to process every two minutes a fragment of a track.

4) The performance and cost summary of this system: Assuming an average density of space debris is $0.2 \mathrm{~g} / \mathrm{cm}^{2}$, eliminate debris $10 \mathrm{~cm}$ diameter monitoring time required for $9 \mathrm{~min}$, laser ablation time was 9 days. We chose to use the lowest cost relative to a laser beam $(=40 \mathrm{~ns}, \mathrm{~W}=20 \mathrm{~kJ}, \mathrm{~F}=1$ $\mathrm{Hz}, \mathrm{P}=20 \mathrm{~kW})$.

Based on this device can be used within four years the average power of $20 \mathrm{kw}$ laser disposed within the diameter of $1000 \mathrm{~km}$ of all debris in $1 \mathrm{~cm} \sim 1 \mathrm{~m}$ above sea level between the total cost of $\$ \$ 2.2032 * 1011$, of which the total cost of the monitoring device is $\$ 20 \mathrm{M}$, the total cost of the correction means is $\$ 10 \mathrm{M}$, the laser device costs $\$ 20 \mathrm{M}$, the cost of emitting a laser pulse beam processing debris is $\$ 10 \sim 20 / \mathrm{g}$.

\section{5) Model analysis}

a) Assumptions: (1) The average density of space debris is $0.2 \mathrm{~g} / \mathrm{cm}^{3}$, the elimination of large debris monitoring time required for $9 \mathrm{~min}$, laser ablation time was 9 days. We chose to use a relatively low cost of the laser beam $(=40 \mathrm{~ns}, \mathrm{~W}=20$ $\mathrm{kJ}, \mathrm{F}=1 \mathrm{~Hz}, \mathrm{P}=20 \mathrm{~kW})^{[1]}$

(2) About 12 minutes to eliminate small pieces ${ }^{[1]}$

(3) Process system operation without failure.

(4) $\mathrm{N}=190 \mathrm{k}$, (small objects, diameter $>=1 \mathrm{~cm}$ ) ( Klinkrad, 2006d p. 96 ).

(5) Because of the low orbit space debris damaging, so space debris models only discuss low-orbit (LEO) situation.

b) Symbol description

$N_{1 l}$ :The total number of large space-debris

$N_{1 s}$ :The total number of small space-debris

$t:$ Time

$x$ : Number of units of laser transmitters/ Costs

c) Model: Small ground-based laser can clear small debris ( diameter $<10 \mathrm{~cm}$ ) and large debris (diameter $<1 \mathrm{~m}$ ), small debris and large debris have their own goals a growth model, therefore, we discuss two cases model. Ground and space-based laser to provide greater energy technology is mature, but because of the atmosphere absorption loss is large, and by geographic location and distance makes it possible to influence the scope of the work space is limited, so take each laser transmitter can work 12 hours a day.

Ground-based laser to clear large space-debris model formula is as follows:

$N_{1 l}=-13.6513+1.0013 e^{0.113 t}-0.1110 x t$
Where in the total number of pieces $N_{1 l}, t$ ( Year ) is time, $X$ is the number of laser transmitters. Without human intervention for the total amount of debris, a growth trend from the previous fitting work determined.
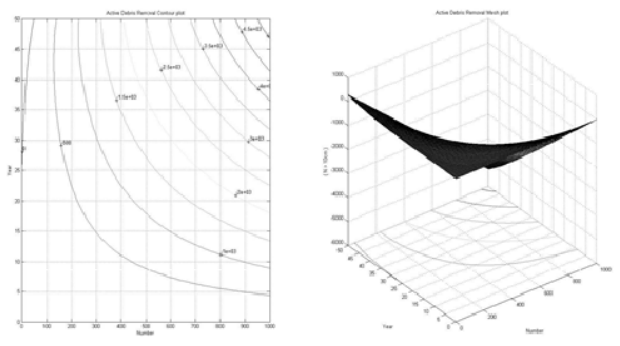

FIGURE IV. GROUND-BASE LASER: EFFECT OF NUMBER OF LASER STATIONS FOR SMALL DEBRIS REMOVED TIME

Ground-based laser to clear small space debris model formula is as follows:

$$
N_{1 s}=190000-78.1617+1.0448 e^{0.0439 t}-60 x t
$$

Where in the total number of pieces $N_{1 s}, t$ ( Year ) is time, $X$ is the number of laser transmitters. Without human intervention for the total amount of debris, a growth trend from the previous fitting work determined.
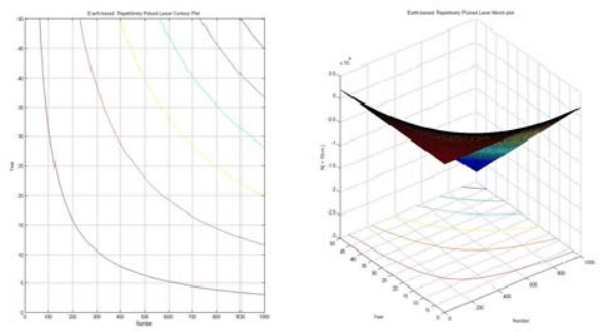

FIGURE V. GROUND-BASE LASER: EFFECT OF NUMBER OF LASER STATIONS FOR LARGE DEBRIS REMOVED TIME

As can be seen from the figure, with the increase of the number of laser device, the rate of reduction of debris increase.

\section{B. Space-Based Repetitively Pulsed Laser}

1)Overview: Space-based high-energy pulse laser two forms of force liquidation LEO debris: one is the light pressure force, similar to sunlight pressure magnitude is very small and can be ignored; the other is acting on the debris laser ablation debris part form gaseous ablation products, leaving the formation of high-speed jets of debris, so the debris to get instantaneous impulse to change the debris orbit. That is, under the platform of maneuvering space-based laser, laser fragments the role of the fragments obtained velocity increment, reducing the perigee height of the orbit of debris orbit until certain height and reach cleanup goals results.

\section{2)Pulse laser effect theory}

Debris could not produce the desired effect of laser interaction energy conversion, $\mathrm{Cm}$ is defined as high-energy 
laser pulse energy acting on the fragments into a single fragment momentum coupling coefficient of variation is: $C_{m}=\frac{m_{0}-\Delta m}{\eta_{c} E_{d}}$ where: $m_{0}$ is debris quality laser action before, $\Delta$ $\mathrm{m}$ is substance mass fragment at the injection laser ablation action, $\eta_{c}$ is lasing efficiency factor, $E_{d}$ is Acting on the debris of a single laser pulse energy. Quality of single nanosecond laser pulse ablation debris is $\Delta m=m \tau$, where $m=-\varepsilon \eta_{c} E_{d}$ is Fragments of mass change rate, $\tau$ is Pulse Width,cis Ablation rate. Suppose the number of high-energy pulsed laser repetition frequency is $\mathrm{N}$, So the average power is $P=N E_{d}$, Single speed laser pulses fragments obtained increment is :

$\Delta v=\frac{\eta_{c} C_{m} p}{[N(m-\Delta m)]}$ Assumed that the laser irradiation energy density (unit effective area of laser energy) is I, S is the cross sectional area irradiation, then $E_{d}=I S$; lasing area of the effective crosssectional area of debris is $S_{D}$, a Gaussian laser beam in space type distribution, the radius of the spot is $d=\frac{4}{\pi}\left(\frac{\lambda R}{D_{a}}\right)$

In this formula, is the laser wavelength; $D_{a}$ is valid beam aperture; $\mathrm{R}$ is the distance between laser generator and debris. So the fragments' speed increment caused by single laser pulses is

$$
\Delta v=\left\{\begin{array}{l}
\eta_{c} C_{m} S_{D} P /\left[N\left(m_{0}-\Delta m\right) \pi d^{2}\right] S_{D}<\pi d^{2} \\
\eta_{c} C_{m} P /\left[N\left(m_{0}-\Delta m\right)\right] S_{D} \geq \pi d^{2}
\end{array}\right.
$$

\section{3)Model analysis}

a) Assumptions . (1) We chose to use a relatively low cost of the laser beam $(=40 \mathrm{~ns}, \mathrm{~W}=20 \mathrm{~kJ}, \mathrm{~F}=1 \mathrm{~Hz}, \mathrm{P}=20$ $\mathrm{kW})[1]$.

(2) Process system operation without failure.

(3) $\mathrm{N}=190 \mathrm{k}$, (small objects, diameter $>=1 \mathrm{~cm}$ ) (Klinkrad, 2006d p. 96 ).

(4) Because of the low orbit space debris damaging, so space debris models only discuss low-orbit (LEO) situation.

(5) Using the best irradiation angle.

b) Symbol Description .

$N_{2 l}$ : The total number of large space-debris

$N_{2 s}$ : The total number of small space-debris

\section{$t$ : Time}

\section{$x:$ Number of units of laser transmitters/ Costs}

c) Model: We same discuss that model of Space-based laser clean up small pieces and large pieces case by case, propagation in a vacuum, and there is no loss negligible refraction error propagation and scattering influence, with the change of platform deployment, scalable control the spatial extent, it is considered that each laser device can work 24 hours a day. The use of space-based laser device, dispose of a $10 \mathrm{~cm}$ debris needs $8385 \mathrm{~s}^{[2]}$, so we believe that each laser device can handle a ten pieces a day, For large debris, Since the space-based laser could not consider the weather and other factors, we thought that space-based laser can handle one large debris a day.

Space-based laser to clear large space debris model formula is as follows:

$N_{2 l}=-13.6513+1.0013 e^{0.113 t}-x t$ where in the total number of pieces $N_{2 l}, t$ (Year) is time, $x$ is the number of laser transmitters. Without human intervention for the total amount of debris, a growth trend from the previous fitting work determined.
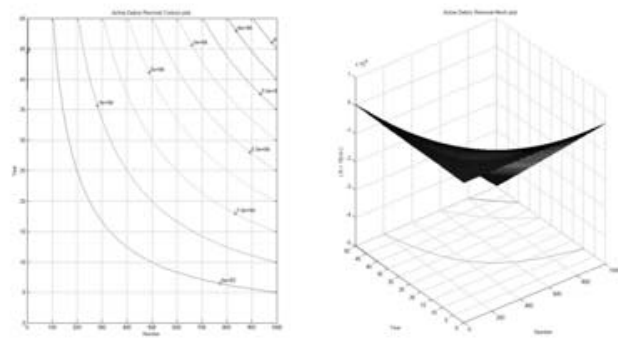

FIGURE VI. SPACE-BASE LASER: EFFECT OF NUMBER OF LASER STATIONS FOR SMALL DEBRIS REMOVED TIME.

Space-based laser to clear small space debris model formula is as follows:

$$
N_{2 s}=190000-78.1617+1.0448 e^{0.0439 t}-10 x t
$$

Where in the total number of pieces $N_{2 s}, t$ ( Year ) is time, $X$ is the number of laser transmitters. Without human intervention for the total amount of debris, a growth trend from the previous fitting work determined.
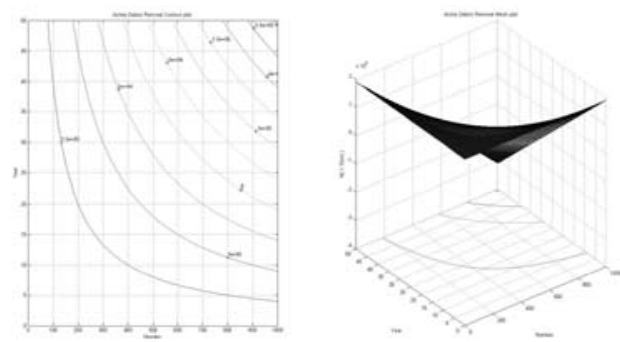

FIGURE VII. SPACE-BASE LASER: EFFECT OF NUMBER OF LASER STATIONS FOR LARGE DEBRIS REMOVED TIME.

\section{Active space Debris Removal Technology}

Space approximately exists 2,200 large debris ( diameter $>=100 \mathrm{~cm})^{[3]}$, Another possible way to clear these large debris is active space debris removal technology. Bonnal has estimated a cost of 27M\$ per large object (Bonnal,2009) for attaching deorbiting kits ${ }^{[3]}$, We will deal with this as a removal satellite debris cost. Due to the high cost of this approach applies only to special cases, so there is no N-t-x model analysis. Given this approach does apply to some 
special cases, We discuss the cost later time will consider this method.

\section{Compare Methods}

Different methods of cost and efficiency are adopted for eliminating space debris for different situations, ground-based laser has to eliminate space debris maximum efficiency, space-based laser has to eliminate large debris maximum efficiency. For large debris, we need to use active space debris removal technology, which is the most one.

\section{COST - RISK MODEL}

\section{A. Proposed Models to the Debris Problem}

A company with the amount of $M$ funds can be used as an investment period. There are 3 methods $\left(S_{i}\right)$ to eliminate cosmic debris can be selected as investment projects, investors can evaluate these three methods', estimated variable costs $\left(r_{i}\right)$,fixed costs $\left(p_{i}\right)$ in this period of time of purchase, the risk of loss rate $\left(q_{i}\right)$ and a method to remove all cosmic debris within a two-year with a fixed cost, as shown in Table 1 , the variable costs and overall risk only affected by $r_{i}, p_{i}, q_{i}$.In the form of fixed costs have been prorated to the variable costs, variable costs can be calculated directly. Now we want to design a portfolio of programs, as large as possible so that the net risk as small as possible.

Assumed that there are $9 \times 10^{9}$ debris need to be cleaned up in LEO:

TABLE I. PORTFOLIO PLAN

\begin{tabular}{|l|l|l|l|}
\hline Investment Project $\boldsymbol{S}_{\boldsymbol{i}}$ & Cost/g & $\begin{array}{l}\text { The risk of } \\
\text { loss rate }\end{array}$ & Transaction Rate \\
\hline Gas solution & 0 & 0 & $800 \mathrm{M}$ \\
\hline Earth - based & 15 & 53 & $8 \mathrm{M}$ \\
\hline Space - based & 20 & 5 & $290 \mathrm{M}$ \\
\hline $\begin{array}{l}\text { Active space debris } \\
\text { removal technology }\end{array}$ & 32 & 11 & 0 \\
\hline
\end{tabular}

\section{B. The Problem Assumptions and Symbol Description}

The overall risk of investment available in the five biggest risks to a measure; In investments, not adjusted for inflation, so the expected cost for the actual variable costs; The system does not consider the risk that the entire market overall risks, it depends on the operation of the economy as a whole as well as unforeseen circumstances that may occur in the universe, investors can not disperse the risk, but only consider unsystematic risk, which investors by investing in the risk of some types of selection dispersed; Do not consider the mental capacity of investors for risk.

\section{Model Analysis}

Fixed costs :

$$
f\left(x_{i}\right)=\left\{\begin{array}{ll}
p_{i} & x_{i}>0 \\
0 & x_{i}=0
\end{array} \quad i=1,2,3\right.
$$

$$
\begin{aligned}
& \text { Variable costs : } \quad R=\sum_{i=1}^{3} r_{i} \times x_{i} \\
& \text { Overall risk : } Q=\sum_{i=1}^{3} r_{i} \times q_{i} \times x_{i} \\
& \text { Restrictions : } \sum_{i=1}^{3} x_{i}=9 \times 10^{6}
\end{aligned}
$$

Neglecting $M$, The original problem can be transferred into a double objective decision making problem.

$$
\min \mathrm{R}=\sum_{i=1}^{3} r_{i} x_{i} x_{i} q_{i} \min \mathrm{Q}=\sum_{i=1}^{3} r_{i} x_{i} q_{i}\left\{\begin{array}{c}
\sum_{i=1}^{3} r_{i} x_{i}=k \\
\sum_{i=1}^{3} x_{i}=9 \times 10^{6} \\
x_{i} \geq 0 \\
i=1,2,3
\end{array}\right.
$$

The solution of the model

(In the following fitting process, to facilitate the calculation, the magnitude suffix is ignored and it does not affect the results)

Minimizing $Q$ with fixed $R$ : The model (3.1) can be transferred into the following model:

$$
\min \mathrm{Q}=\sum_{i=1}^{3} r_{i} x_{i} q_{i} .\left\{\begin{array}{c}
\sum_{i=1}^{3} r_{i} x_{i}=k \\
\sum_{i=1}^{3} x_{i}=9 \times 10^{6} \\
x_{i} \geq 0 \\
i=1,2,3
\end{array}\right.
$$

This model can be rewrite as the following formula :

$\min y \quad$ s.t. $\left\{\begin{array}{c}r_{1} x_{1}+r_{2} x_{2}+r_{3} x_{3}=R \\ x_{1}+x_{2}+x_{3}=9 \times 10^{6} \\ x_{i} \geq 0, y_{i} \geq 0 \\ i=1,2,3\end{array}\right.$

The Earth-based laser needs $\$ 2.2032 \times 10^{11}$ to remove all debris;

The Space-based laser needs $\$ 4.935 \times 10^{11}$ to remove all debris;

The Gas solution needs $\$ 3.1968 \times 10^{11}$ to remove all debris.

In fact, it can be calculated by Lingo to get a result as followed:

TABLE II. THE MODEL RESULTS

\begin{tabular}{|l|l|l|l|l|}
\hline $\begin{array}{l}\text { Cost } \\
\mathbf{R} / 10^{8}\end{array}$ & \multicolumn{1}{|c|}{$\begin{array}{c}\text { Minimum risk rate } \\
\mathbf{Q} / 10^{6}\end{array}$} & $X_{0}$ & $X_{1}$ & $X_{2}$ \\
\hline 2.3 & 19.54167 & 4.8333 & 0 & 4.1667 \\
\hline 2.4 & 21.65000 & 4.0000 & 0 & 5.000 \\
\hline 2.5 & 23.75833 & 3.1667 & 0 & 5.8333 \\
\hline 2.6 & 25.86670 & 2.3333 & 0 & 6.6667 \\
\hline 2.7 & 27.97500 & 1.5000 & 0 & 7.5000 \\
\hline 2.9 & 31.90000 & 0 & 0 & 9.0625 \\
\hline 3.1 & 34.10000 & 0 & 0 & 9.6875 \\
\hline 3.3 & 36.30000 & 0 & 0 & 10.3125 \\
\hline 3.5 & 38.50000 & 0 & 0 & 10.9375 \\
\hline 3.7 & 40.70000 & 0 & 0 & 11.5625 \\
\hline 3.9 & 42.90000 & 0 & 0 & 12.1875 \\
\hline
\end{tabular}


According to the fitting results, a relationship between minimum risk and the cost of change can be got with fixed cost.

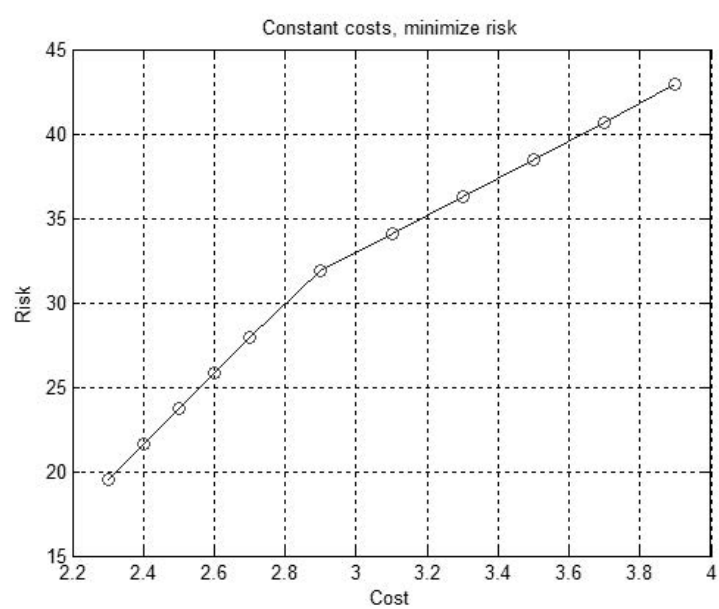

FIGURE VIII. RELATIONSHIP BETWEEN VARIABLE COSTS AND RISKS

As can be seen from the figure, with the growth of variable costs, the minimum risk is an increasing trend, which is due to the elimination of cosmic debris that industry is a relatively small minority of the industry, the market price is relatively fixed, increased costs, meaning increased investment, which means an increase in income, so the higher the income, the greater the risk, but after 2.9, with the risk of increased earnings trend is clearly slowing down, the investment is relatively cost-effective.

\section{Minimizing $R$ with Fixed $Q$.}

The model (3.1) can be transferred into the following model: $\min \mathrm{R}=\sum_{i=1}^{3} r_{i} x_{i} \quad \mathrm{Q}=\sum_{i=1}^{3} r_{i} x_{i} q_{i} \quad \sum_{i=1}^{3} x_{i}=9 \times 10^{6}$

The parameters of risk:

1. The Earth-based laser: $9 \times 10^{6}$

2.The Space-based laser: $71.55 \times 10^{6}$

3.The Gas solution: $31.68 \times 10^{6}$

$$
\min \mathrm{R}=\sum_{i=1}^{3} r_{i} x_{i} \text { s.t. }\left\{\begin{array}{c}
\sum_{i=1}^{3} r_{i} x_{i} q_{i}=R Q \\
\sum_{i=1}^{3} x_{i}=9 \times 10^{6} \\
x_{i} \geq 0 \\
i=1,2,3
\end{array}\right.
$$

MATLAB program was compiled, start with the smallest risk of $\mathrm{Q}=9$, for each increase of 5 percentage points of risk search ${ }^{[5]}$. Calculated according to the correspondence between the minimum cost and minimum risk and investment allocation between the calculated results are tabulated as following figure:
TABLE III.

THE MODEL RESULTS

\begin{tabular}{|c|c|c|c|c|}
\hline Risk $\boldsymbol{Q}$ & Variable cost $\boldsymbol{R}$ & $\boldsymbol{X}_{0}$ & $\boldsymbol{X}_{1}$ & $\boldsymbol{X}_{2}$ \\
\hline 9 & 18 & 9 & 0 & 0 \\
\hline 10 & 17.92806 & 8.8561 & 0.1439 & 0 \\
\hline 15 & 17.56835 & 8.1367 & 0.8633 & 0 \\
\hline 20 & 17.2086 & 7.4172 & 1.5827 & 0 \\
\hline 25 & 16.8489 & 6.6978 & 2.3021 & 0 \\
\hline 30 & 16.4892 & 5.9784 & 3.0216 & 0 \\
\hline 35 & 16.1295 & 5.2590 & 3.7410 & 0 \\
\hline 40 & 15.7698 & 4.5396 & 4.4604 & 0 \\
\hline 45 & 15.0504 & 3.1007 & 5.8993 & 0 \\
\hline 50 & 15.0504 & 3.1007 & 5.8993 & 0 \\
\hline 55 & 14.6906 & 2.3812 & 6.6187 & 0 \\
\hline 60 & 14.3309 & 1.6618 & 7.3381 & 0 \\
\hline 65 & 13.9712 & 0.9424 & 8.0576 & 0 \\
\hline 70 & 13.6115 & 0.2230 & 8.7770 & 0 \\
\hline 71.55 & 13.5 & 0 & 9 & 0 \\
\hline
\end{tabular}

As can be seen from the table, under the risk is known, the higher the risk, the lower the variable costs. When you want to receive the same benefits, the less the cost of inputs, the greater the need to withstand risks, can be seen from the figure about the risks and costs is a negative linear correlation between risk and 40 to 50 , there are two by correlation of greater volatility, the slope between 40 to 45 increased significantly, there is a big opportunity, but 45 to 50 is not suitable for investment.

\section{The Model of Minimize QR}

According to Profit- Maximum Risk Principle, a model can be built as following:

$\min \mathrm{Q}=R Q \quad$ s.t. $\left\{\begin{array}{c}\sum_{i=0}^{5}\left(1+p_{i}\right) x_{i}=1 \\ x_{i} \geq 0(i=0,1, \mathrm{~L}, 5)\end{array}\right.$

While $q_{0}=0, x_{0}=9 \times 10^{6}, x_{1}=x_{2}=\mathrm{L}=0, \min R Q=0$

For this issue, the rate of return and risk of loss will not affect the other investments the optimal solution, so this model is not good enough.

\section{EXECUTIVE SUMMARY}

Four methods are chosen in our model: Clearing nearEarth space debris using an Earth-based, repetitively pulsed laser, Clearing near-Earth space debris using an Space-based laser, Active space debris removal technology 1 and Gas solution. After learning the four methods in detail, an assessment was made to compare the cost and risk of the four methods and the combination of them.

According to the analysis, without human intervention, development of space debris is exponential growth. Different methods of cost and efficiency alone eliminate space debris is different, ground-based laser to eliminate small space debris(in $1 \mathrm{~cm}-10 \mathrm{~cm}$ size) has the maximum efficiency, space-based laser to eliminate large debris (in $10 \mathrm{~cm}-100 \mathrm{~cm}$ size)has the maximum efficiency, For extreme large debris, we need to use 
active space debris removal technology, Which is the most expensive one.

We established a Cost-Risk model to analyze and compare the four methods and found that the Earth-based laser method has the least total cost and the highest risk factor. Taking the risk factors and benefits into account, we analyze and optimized the model and got a conclusion that under different conditions, we need to choose different methods or combinations of those four methods to remove the debris effectively.

\section{REFERENCES}

[1] C. R. Phipps, \&C. Ho, \&W. Priedhorsky, \& M. M. Michaelis, \&J. P Reilly, (1966), Clearing near-Earth space debris using a 20-kW, 530-nm, Earth-based, repetitively pulsed laser, Present address: Photonic Associates, 1621 Calle Torreon, Santa Fe, NM 87501.

[2] Han W H, \&Gan Q B, \&He Y, et al. Optimal direction and a process design of removing low Earth orbit debris with space-based laser[J]. Acta Aeronautica et Astronautica Sinica, 2015, 36(3):749-756.

[3] Claude R. Phipps, \& Kevin L. Baker, \&Stephen B. Libby et al(2012).Removing orbital debris with lasers, Advances in Space Research 49 (2012) 1283-1300.

[4] Klinkrad, H. Space Debris -Models and Risk Analysis, Praxis Publishing, Chichester, UK, pp. 96, 2006d.

[5] ZHU Yi-lin, Chinese Academy of Space Technology, Beijing 100081, China, modelling and analysing of space debris environment in low earth orbit,aerospace shanghai: 1006-1630(2000) 03-0018-05.

[6] J.-C. Liou *, Collision activities in the future orbital debris environment, Lockheed Martin Space Operations, Mail Code C104, 2400 NASA Parkway, Houston, TX 77058, USA, Advances in Space Research 38 (2006) 2102-2106.

[7] J.-C. Liou ,* et al., LEGEND - a three-dimensional LEO-to-GEO debris evolutionary model, Lockheed Martin Space Operations, 2400 NASA Road 1, Mail Code C104, Houston, TX 77058, USA, Advances in Space Research 34 (2004) 981-986. 